\title{
Effect of Composition on the Redox Performance of Strontium Ferrite Nanocomposite
}

Wenbo $\mathrm{Yu}^{1 \ddagger}$, Xinhe Wang ${ }^{2 \ddagger}$, Yanshou Liu ${ }^{1}$, Jinjia Wei ${ }^{1,2}$, Junshe Zhang, ${ }^{1, *}$

${ }^{1}$ School of Chemical Engineering and Technology, Xi'an Jiaotong University, Xi'an, Shaanxi 710049, China

${ }^{2}$ State Key Laboratory of Multiphase Flow in Power Engineering, Xi'an Jiaotong University, Xi'an, Shaanxi 710049, China

$\ddagger$ First authors

Figure S1. Elution profiles of gaseous products in $\mathrm{CH}_{4}-\mathrm{POx}$ over the quartz wool (a) and $\mathrm{CaO}(\mathrm{b})(m=0.5 \mathrm{~g}): T=980^{\circ} \mathrm{C}, P=1 \mathrm{~atm}, F_{\text {red }}=50 \mathrm{STP} \mathrm{mL} \mathrm{min}{ }^{-1}\left(4 \mathrm{vol} . \% \mathrm{CH}_{4}\right)$.

Figure S2. Equilibrium $\mathrm{CO} 2$ pressure of $\mathrm{CaCO} 3$ decomposition as a function of temperature.

Figure S3. Elution profiles of gaseous products in $\mathrm{CH}_{4}-\mathrm{POx}$ and $\mathrm{CO}_{2}$-splitting of the $3^{\text {rd }}$ cycle of SFO $(m=0.13 \mathrm{~g}): T=980^{\circ} \mathrm{C}, P=1 \mathrm{~atm}, F_{\text {red }}=50 \mathrm{STP} \mathrm{mL} \min ^{-1}(4 \mathrm{vol} . \%$ $\left.\mathrm{CH}_{4}\right), F_{\text {ox }}=100 \mathrm{STP} \mathrm{mL} / \mathrm{min}\left(5\right.$ vol. $\left.\% \mathrm{CO}_{2}\right)$.

Table S1. Redox performance of SFOC-3 at 900, 950 and $950{ }^{\circ} \mathrm{C}$.

Table S2. Lattice parameters of $\mathrm{SrFeO} 3-\delta$ (Tetragonal) and $\mathrm{CaO}$ (Cubic) in four nanocomposites and pure phases. 

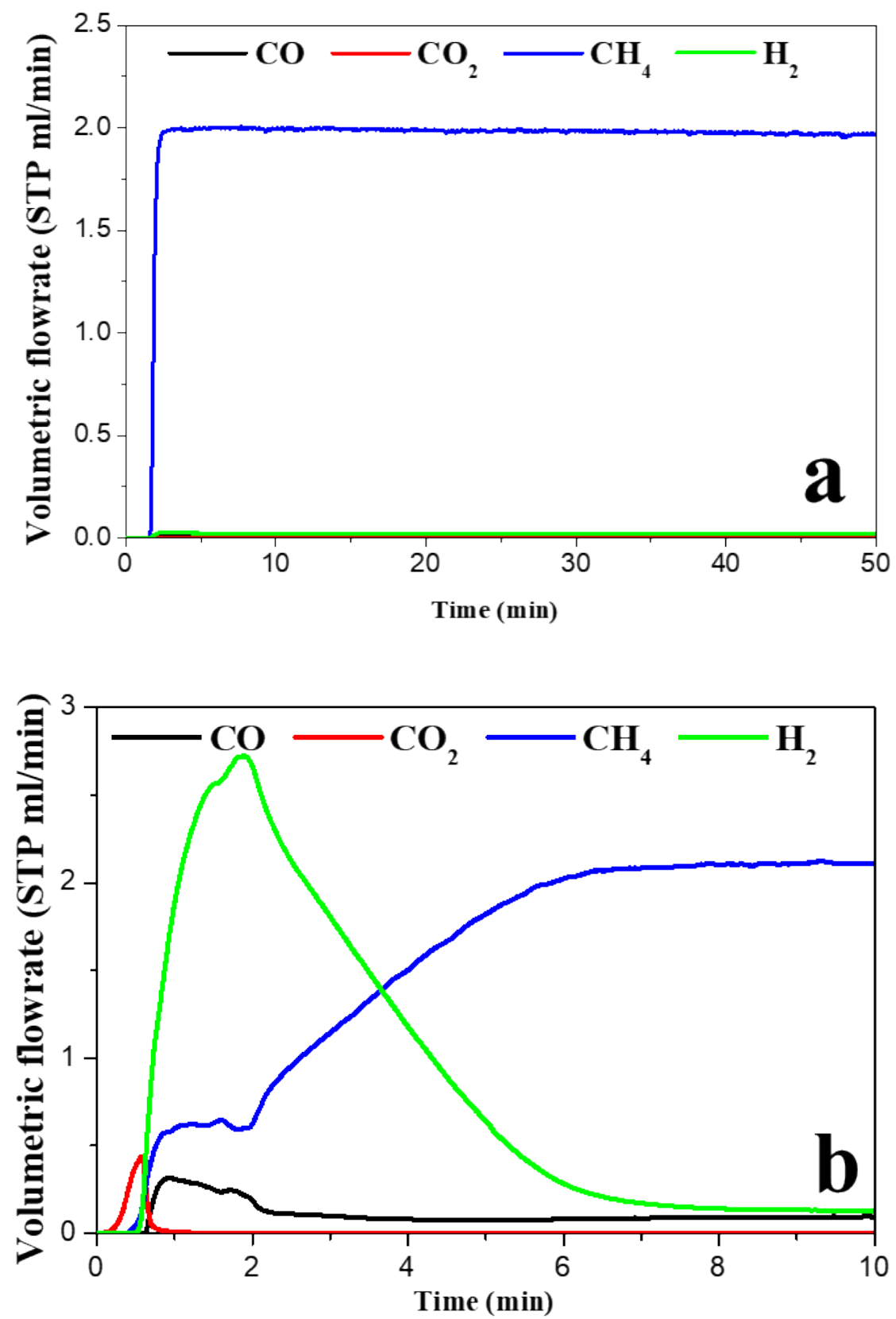

Figure S1. Elution profiles of gaseous products in $\mathrm{CH}_{4}-\mathrm{POx}$ over the quartz wool (a) and $\mathrm{CaO}(\mathrm{b})(m=0.5 \mathrm{~g}): T=980^{\circ} \mathrm{C}, P=1 \mathrm{~atm}, F_{\mathrm{red}}=50 \mathrm{STP} \mathrm{mL} \min ^{-1}\left(4 \mathrm{vol} . \% \mathrm{CH}_{4}\right)$. 


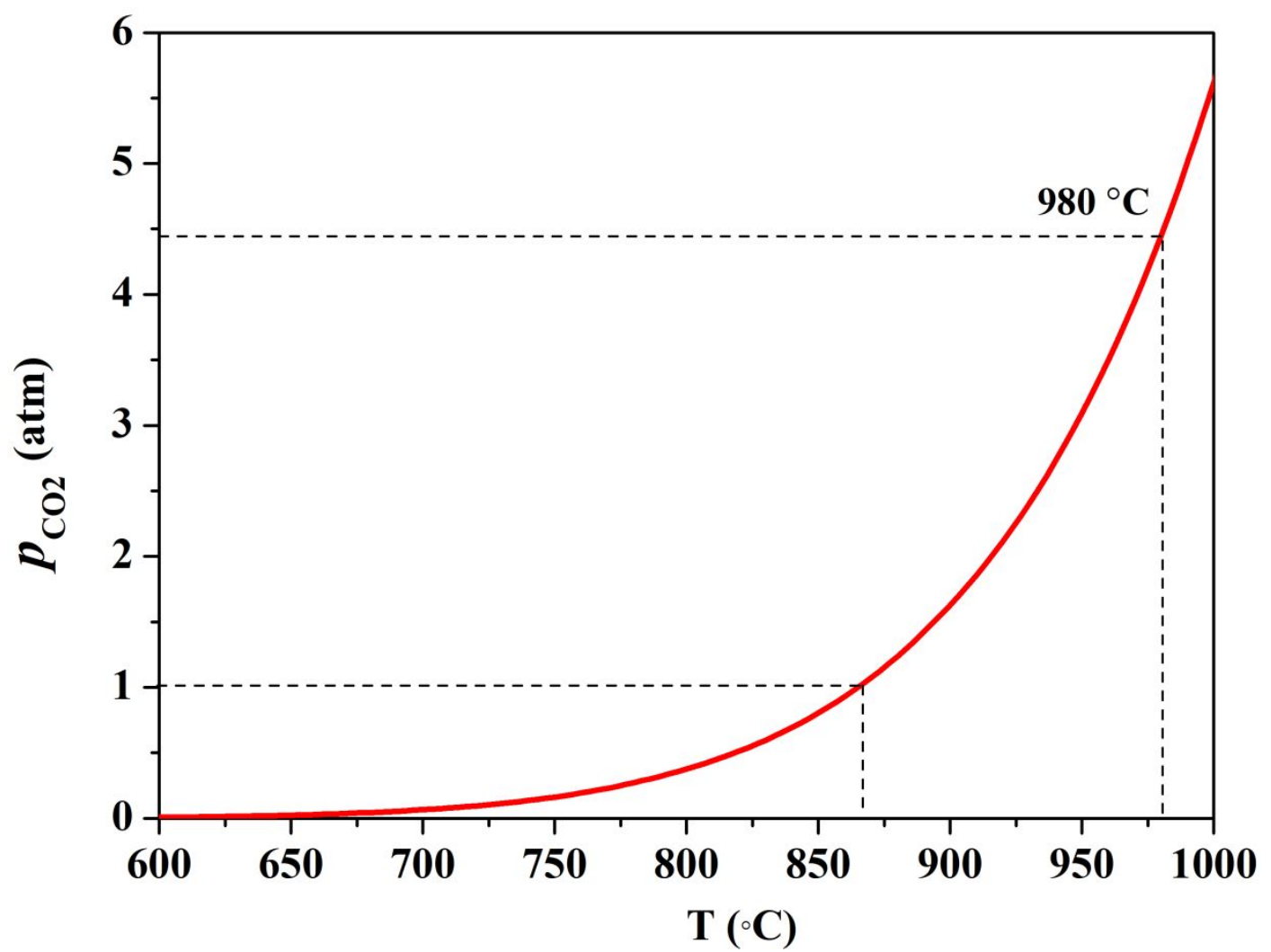

Figure S2. Equilibrium $\mathrm{CO}_{2}$ pressure of $\mathrm{CaCO}_{3}$ decomposition as a function of temperature. 

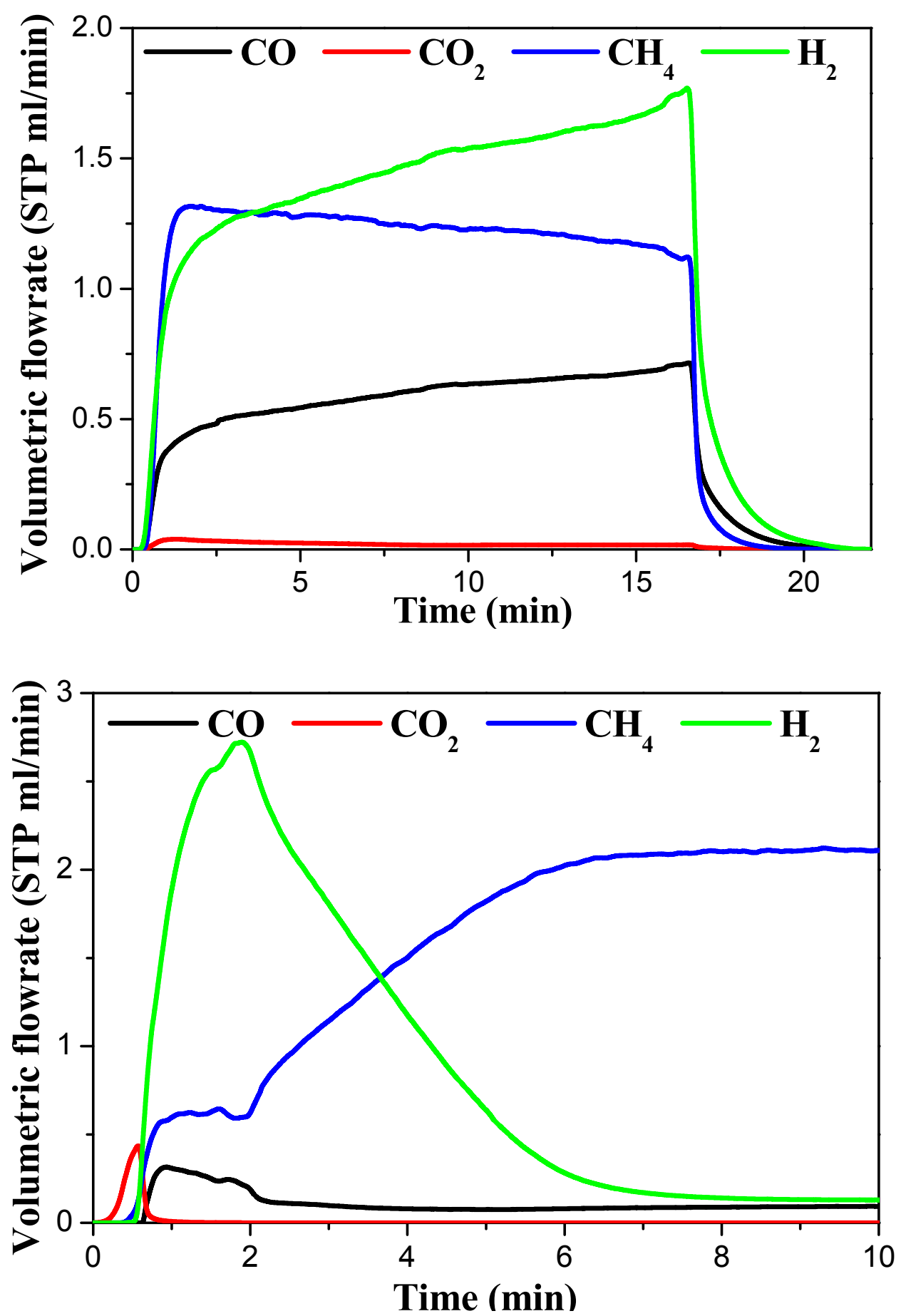

Figure S3. Elution profiles of gaseous products in $\mathrm{CH}_{4}-\mathrm{POx}$ and $\mathrm{CO}_{2}$-splitting of the $3^{\text {rd }}$ cycle of SFO $(m=0.13 \mathrm{~g}): T=980^{\circ} \mathrm{C}, P=1 \mathrm{~atm}, F_{\text {red }}=50 \mathrm{STP} \mathrm{mL} \mathrm{min}^{-1}(4 \mathrm{vol} . \%$ $\left.\mathrm{CH}_{4}\right), F_{\text {ox }}=100 \mathrm{STP} \mathrm{mL} / \mathrm{min}\left(5 \mathrm{vol} . \% \mathrm{CO}_{2}\right)$. 
Table S1. Redox performance of SFOC-3 at 900,950 and $950^{\circ} \mathrm{C}^{\dagger}$

\begin{tabular}{llll}
\hline & $900{ }^{\circ} \mathrm{C}$ & $950{ }^{\circ} \mathrm{C}$ & $980{ }^{\circ} \mathrm{C}$ \\
\hline$t_{\mathrm{R}}(\min )$ & 14.2 & 9.4 & 7.9 \\
$\mathrm{CH}_{4}$ conversion (\%) & 48 & 60 & 64 \\
Syngas selectivity (\%) & 88 & 90 & 90 \\
Coke selectivity (\%) & 2.6 & 2.6 & 3.0 \\
Syngas productivity $\left(\mathrm{mol} \cdot \mathrm{kg}_{\mathrm{SFO}}{ }^{-1}\right)$ & 5.8 & 8.5 & 9.6 \\
\hline treaction conditions: $m_{\mathrm{SFOC}-3}=0.43 \mathrm{~g}, P=1 \mathrm{~atm}, F_{\text {red }}=50 \mathrm{STP} \mathrm{mL} / \mathrm{min}\left(4 \mathrm{vol} . \% \mathrm{CH}_{4}\right)$.
\end{tabular}

Table S2. Lattice parameters of $\mathrm{SrFeO}_{3-\delta}$ (Tetragonal) and $\mathrm{CaO}$ (Cubic) in four nanocomposites and pure phases

\begin{tabular}{llrrrrr}
\hline & \multicolumn{5}{c}{ Parameters $(\AA)$} \\
\cline { 2 - 7 } & $a$ & 10.934 & SFOC-1 & SFOC-3 & SFOC-6 & SFOC-8 \\
\hline \multirow{3}{*}{$\mathrm{SrFeO}_{3-\delta}$} & $b$ & 10.934 & 10.95227 & 10.93270 & 10.93941 & 10.93949 \\
& $c$ & 7.705 & 10.95227 & 10.93270 & 10.93941 & 10.93949 \\
& $a$ & & 7.68900 & 7.68684 & 7.71826 & 7.69202 \\
$\mathrm{CaO}$ & $b$ & 4.81059 & 4.81944 & 4.82330 & 4.83736 & 4.83175 \\
& $c$ & 4.81059 & 4.81944 & 4.82330 & 4.83736 & 4.83175 \\
& & 4.81059 & 4.81944 & 4.82330 & 4.83736 & 4.83175 \\
\hline
\end{tabular}

